\title{
Diâmetro Mínimo e Ciclo de Corte de Qualea paraensis Duke no Norte do Mato Grosso, Brasil
}

\section{Minimum diameter and cutting cycle of Qualea paraensis Duke in North of Mato Grosso, Brazil}

Ana Paula Zopeletto Massing ${ }^{1}$, Dirceu Lúcio Carneido de Miranda ${ }^{2 *}$, Gerson dos Santos Lisboa $^{3}$, Luciano Cavalcante de Jesus França ${ }^{4}$, Thiago Floriani Stepka ${ }^{5}$, Thamires Oliveira da Silva $^{6}$, Tiago Monteiro Condé ${ }^{7}$, Luciano Farinha Watzlawick ${ }^{8}$

\begin{abstract}
RESUMO
O manejo florestal é regulamentado pela Instrução Normativa ${ }^{\circ} 5$ de 11/12/2006, a qual é estabelecida um Diâmetro Mínimo de Corte (DMC) de $50 \mathrm{~cm}$ e um Ciclo de Corte (CC) de 25 a 35 anos, para toda as espécies florestais, desconsiderando as variações do crescimento de cada espécie na floresta. Os objetivos desse estudo foi descrever o crescimento de Qualea paraensis, estimar o DMC e o CC da espécie. No município de Santa Carmem (MT), Brasil, foram coletadas 19 unidades amostrais (árvores) de forma destrutiva, as quais foram secas em temperatura ambiente e submetidas ao processo de polimento. Para cada árvore foi marcado e digitalizado dois raios e mensurado os anéis de crescimento. A partir disso, foram construídas as curvas de crescimento em função da idade para cada indivíduo amostrado. Para estimar o DMC e o CC utilizou-se a modelagem de crescimento com base em três funções não lineares: Gompertz, Ratowsky e Weibull. A idade média dos indivíduos foi de 68 anos e o diâmetro médio foi de $49 \mathrm{~cm}$. Q. paraensis apresentou um alto incremento corrente anual médio de $0,70 \mathrm{~cm}$.ano ${ }^{-1}( \pm 0,11)$. A espécie leva em média 87 anos para atingir o incremento em volume. Estimou-se o DMC de aproximadamente $49 \mathrm{~cm}$, e o CC de 17 anos. A legislação atual pode superestimar ou subestimar o ritmo de crescimento tornando o manejo florestal muitas vezes insustentável, contudo, estas práticas devem ser aliadas a estudos em longo prazo de dinâmica florestal.
\end{abstract}

Palavras-chave: Anéis de Crescimento. Cambará. Dendrocronologia. Manejo Florestal.

\begin{abstract}
Forest management is regulated by Normative Instruction IN 05 of 11/12/2006, which establishes a Minimum Cutting Diameter (MCD) of $50 \mathrm{~cm}$, a Cutting Cycle (CC) from 25 to 35 years, for all species forestry, disregarding the variations in the growth of each species in the forest. The objectives of this research are to describe the growth of Q. paraensis, estimate the MCD and CC of the species. In the
\end{abstract}

\footnotetext{
${ }^{1}$ Universidade Federal de Mato Grosso (UFMT) Mato Grosso, Brasil.

${ }^{2}$ Universidade Federal de Mato Grosso (UFMT), Mato Grosso, Brasil.

${ }^{3}$ Universidade Federal de Goiás (UFG), Goiás, Brasil.

${ }^{4}$ Instituto Federal de Minas Gerais (IFMG), Minas Gerais, Brasil.

${ }^{5}$ Universidade do Estado de Santa Catarina (UDESC), Santa Catarina, Brasil.

${ }^{6}$ Universidade Federal do Sul da Bahia (UFSB), Bahia, Brasil.

${ }^{7}$ Universidade Estadual de Roraima (UERR), Roraima, Brasil.

${ }^{8}$ Universidade Estadual do Centro Oeste (UNICENTRO), Paraná, Brasil.

*Autor correspondente: mirandaufpr@yahoo.com.br
} 
municipality of Santa Carmem-MT, Brazil, 19 sampling units (trees) were collected in a destructive way, which were dried at room temperature and submitted to the polishing process. For each tree, two rays were marked and digitized and the growth rings measured. From this, growth curves were constructed as a function of age for each sampled individual. To estimate the MCD and CC, growth modeling based on three nonlinear functions was used: Gompertz, Ratowsky and Weibull. The mean age of individuals was 68 years and the mean diameter was $49 \mathrm{~cm}$. Q. paraensis presented a high mean annual current increment of 0.70 cm.year ${ }^{-1}( \pm 0.11)$. The species takes an average of 87 years to reach the increase in volume. The CMD of approximately $49 \mathrm{~cm}$ and the $\mathrm{CC}$ of 17 years were estimated. Current legislation can overestimate or underestimate the rate of growth, making forest management often unsustainable, however, these practices must be combined with long-term studies of forest dynamics.

Keywords: Amazon. Growth Rings. Cambara. Dendrocronology. Forest Management.

\section{INTRODUÇÃO}

No bioma Amazônico, muitas florestas nativas estão entrando em um segundo ciclo de corte (MIRANDA et al., 2018). Contudo, ainda existem lacunas de estudos que possibilitem a melhoria de aproveitamento dos recursos florestais de forma sustentável. Uma das melhores formas de manter a floresta em pé e manter seus serviços ecossistêmicos é por meio do manejo florestal (GROENENDIJK et al., 2014). O manejo florestal sustentável trata-se de um conjunto de princípios, técnicas e normas, que tem a finalidade de estabelecer as ações necessárias para ordenar os fatores de produção e controlar a sua produtividade e eficiência para alcançar objetivos pré-definidos (HIGUCHI, 1994).

Atualmente, o manejo florestal na Amazônia brasileira, é regulamentado pela Instrução Normativa $n^{\circ} 05$ de 11/12/2006 do Ministério do Meio Ambiente, a qual é estabelecido um sistema policíclico, Diâmetro Mínimo de Corte (DMC) de $50 \mathrm{~cm}$, um Ciclo de Corte (CC) de 25 a 35 anos, e remoção do volume de até $30 \mathrm{~m}^{3}$.ha-1, para todas as espécies florestais, assim desconsiderando as variações do incremento diamétrico por espécie da floresta. Entretanto, é permitido o estabelecimento de um DMC e CC específicos por espécie, com base em estudos técnicos e ecológicos. No Amazonas, por exemplo, foram estabelecidos DMCs e CCs para espécies alagáveis (SCHÖNGART, 2008). Para que se atinja sucesso no manejo florestal, é necessária a sustentabilidade ecológica da produção da madeira que requer informações sobre as variações de crescimento das espécies comerciais, os quais ainda não são utilizados com frequência em florestas tropicais. 
No manejo florestal, a obtenção de dados confiáveis sobre o crescimento das árvores, é um dos grandes desafios, pois são informações de suma importância para determinar os volumes de madeira e os ciclos de corte futuros (ROSA et al., 2017). A dendrocronologia, ciência que analisa os anéis de crescimento das árvores, gera informações sobre as variações de crescimento radial, relacionando-as com fatores ambientais (WORBES, 2004). Esses estudos surgem como uma ferramenta de respostas rápidas que possibilitam viabilizar os estudos sobre o crescimento da floresta, que serve de base para o manejo florestal. Em florestas tropicais ainda é incipiente os estudos que tratam do crescimento dessas florestas utilizando dendrocronologia, devido à dificuldade de visualização dos anéis de crescimento. A sazonalidade climática, por exemplo, é um dos fatores responsáveis pela formação e consequentemente a visualização dos anéis de crescimento. É necessária uma estação seca anual com um comprimento de 2 a 4 meses e menos que $60 \mathrm{~mm}$ de precipitação mensal para induzir anéis anuais (WORBES, 1995). Diante deste contexto, a sazonalidade marcante com relação a precipitação pode ser o fator determinante para a formação dos anéis de crescimento nas florestas tropicais de terra firme.

Apesar de constarem aproximadamente 50 espécies com potenciais madeireiros, a base regional da área de estudo variou entre 12 e 24 espécies comerciais (CIPEM, 2012). Dessa forma, o foco principal para estudos de manejo, devem ser voltados a estas espécies desejadas economicamente. Dentre essas espécies, destaca-se Cambará (Qualea paraensis Duke) da família (Vochysiaceae), por ser uma madeira de alta densidade $(0,78 \mathrm{~g} \mathrm{~cm}-3)$, utilizada para diversos fins, incluindo a construção civil (LORENZI, 2002). Em 2015, foi considerada uma das espécies que apresentou o maior volume e valores comercializados pelo Estado de Mato Grosso (SEMA, 2016). Apesar de sua imporância econômica, estudos de modelagem do crescimento e produção para a espécie ainda são escassos.

Recentemente, no Sul do Estado de Mato Grosso, Miranda et al., (2018) utilizaram a dendrocronologia para avaliar o crescimento e determinar o DMC e o CC para Q. paraensis em floresta tropical de terra firme. Entretanto, sabe-se que o crescimento é afetado por fenômenos climáticos, isto é, precipitação, temperatura e luz, bem como, fatores edáficos, como composição química e a estrutura do solo. Desta forma, busca-se saber se esses valores poderiam ser extrapolados para outras regiões do Estado.

Baseado nestes questionamentos e evidências para o avanço do manejo florestal, justifica-se a aplicação da dendrocronologia bem como ferramentas de predição, como 
por exemplo a modelagem do crescimento para avaliar o comportamento do crescimento de Q. paraensis em regiões do Estado de MT. Diante disso, os objetivos dessa pesquisa foram descrever o crescimento de Q. paraensis, e estimar o DMC e o CC.

\section{MÉTODOS}

\section{Área de estudo}

As amostras foram coletadas no Município de Santa Carmem (MT), microrregião

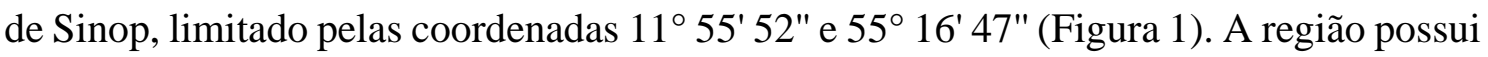
clima tropical quente úmido, temperatura média anual de $24^{\circ} \mathrm{C}$, precipitação anual de $2.000 \mathrm{~mm}$ (VILANI et al., 2006) e sazonalidade climática bem definida, com período de seis meses de déficit hídrico (SOUZA et al., 2013). A área é considerada um ecótono, por tratar-se de uma transição ecológica entre os biomas Cerrado e a Floresta Amazônica, predominando a vegetação nativa com características de Floresta Estacional Semidecidual (ARAUJO et al., 2009).

Figura 1. Mapa de localização do Município de Santa Carmem-MT, Brasil.

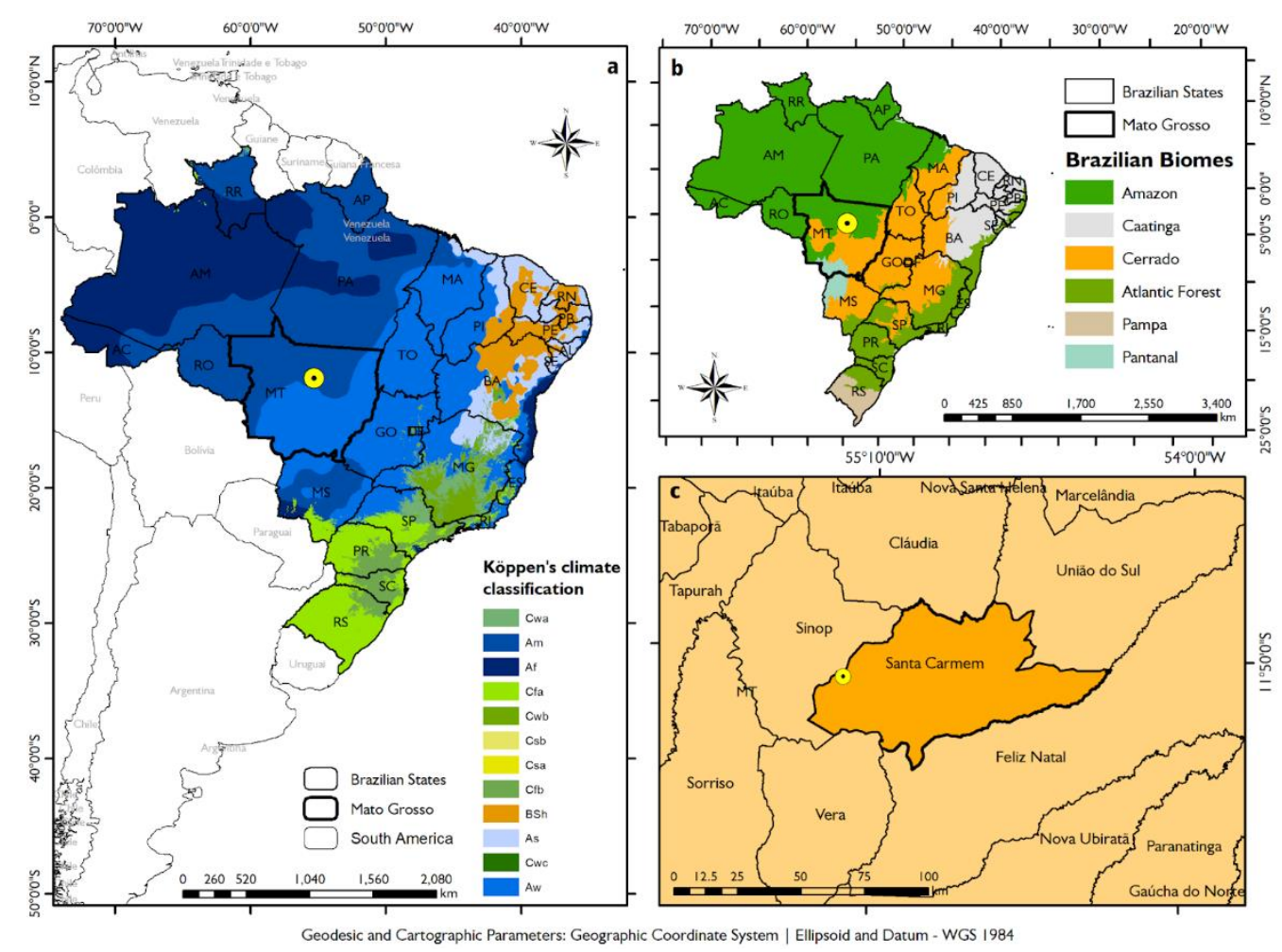

Fonte: Os autores (2022).

Espécie de estudo 
Qualea paraensis, conhecida popularmente como 'cambará', é uma espécie da família Vochysiaceae, com distribuição em toda Amazônia. Segundo Lorenzi (2002), é uma árvore com altura de até 35 metros com tronco reto e cilíndrico e sua madeira é considerada pesada $\left(0,78 \mathrm{~g} / \mathrm{cm}^{3}\right)$ de textura dura e grosseira. É uma espécie muito explorada no Mato Grosso, utilizada para diversos fins comerciais. O principal critério para escolha da espécie foi por apresentar anéis de crescimento visíveis bem demarcados e anuais, conforme descrito por Miranda et al. (2018), além da alta oferta e demanda de exploração madeireira, e por ser de habitat natural de mata de terra firme.

\section{Preparo das amostras, demarcação e mensuração dos anéis de crescimento}

Foram selecionadas 19 árvores as quais foram derrubadas e retirados os discos de madeira de Q. paraensis Duke (a 1,30 m a partir do nível do solo - dap), os quais foram submetidos à secagem em temperatura ambiente, no Laboratório de Manejo Florestal na Universidade Federal de Mato Grosso, Campus de Sinop. Posteriormente uma de suas seções transversais passaram por um processo de polimento, em lixas de diferentes gramaturas (de 36 até 320 grãos $/ \mathrm{mm}^{2}$ ), proporcionando melhor visualização dos anéis de crescimento. A demarcação do limite dos anéis de crescimento foi realizada a olho nu e/ou com auxílio de uma lupa de aumento, delimitando dois raios por amostra, evitando partes com anormalidade do lenho. Contagem do número de anéis de cada raio, e os falsos anéis foram eliminados.

Em seguida, as amostras foram digitalizadas em um Scanner com resolução de 1200 dpi. As larguras dos anéis foram mensuradas, por meio do programa Image Pro Plus ${ }^{\circledR}$ de com precisão de $0,01 \mathrm{~mm}$, no sentido medula- casca. Os dados obtidos por meio do programa foram exportados para o Software Microsoft Excel®.

\section{Determinação e construção das curvas de crescimento}

Para cada ano de vida da árvore foram utilizados os dados de incremento (largura dos anéis) em diâmetro, obtidos pela média dos dois raios de cada amostra, multiplicado por dois. Além disso, foi calculado um fator de correção de diâmetro para cada amostra, devido a mensuração dos raios em partes irregulares, como em discos com a medula deslocada. Os crescimentos em diâmetro da espécie foram descritos graficamente pelo Incremento Médio Anual (IMA) e do Incremento Corrente Anual (ICA) ao longo do tempo. A curva do crescimento acumulado é formada pela soma dos incrementos anuais. A partir desses dados, foram determinadas as possíveis variações e trajetórias de crescimento da espécie, em função da idade de cada árvores amostrada. 


\section{Modelagem de crescimento}

Para estimar o Diâmetro Mínimo de Corte e o Ciclo de Corte de Q. paraensis, utilizou-se a modelagem de crescimento. Foram ajustados por regressão não linear (Tabela 1), as equações sigmoidais Gompertz (1), Ratkowsky (2) e Weibull (3), em que a variável preditora $\mathrm{X}$ é a idade da árvore e a Y, as variáveis de crescimento. Para a seleção do modelo de crescimento, foram considerados os critérios de Coeficiente de Determinação Ajustado $\left(\mathrm{Raj}^{2}\right)$ e o Erro Padrão da Estimativa (Syx).

Tabela 1. Modelos testados para o crescimento do diâmetro e volume, em função da idade.

\begin{tabular}{ccc}
\hline Equação & Modelo & Autor \\
\hline Equação (1) & $\mathrm{y}=\mathrm{a}\left(\mathrm{e}^{-\mathrm{e}^{\mathrm{b}-\mathrm{cx}}}\right)$ & Gompertz \\
Equação (2) & $\mathrm{y}=\frac{\mathrm{a}}{\left(1+\mathrm{e}^{\mathrm{b}-\mathrm{cx}}\right)}$ & Ratkowsky \\
Equação (3) & $\mathrm{y}=\left(\mathrm{a}-\mathrm{be}^{-\mathrm{cx}}\right)$ & Weibull \\
\hline
\end{tabular}

Em que: $\mathrm{y}=$ Incremento em diâmetro ou volume; $\mathrm{a}, \mathrm{b}, \mathrm{c}, \mathrm{d}=$ Coeficientes dos modelos; $\mathrm{e}=$ constante exponencial cuja base é o número de Euler 2,718281828; x = Idade em anos.

Os volumes cumulativos em cada idade foram calculados conforme a equação (4), aprovada para região amazônica do Estado de Mato Grosso, descrita por Colpini, et al. (2009).

$$
\ln (\mathrm{v})=-7,9906+2,241 \ln (\mathrm{dap})
$$

Em que: $\ln (v)=$ logarítmico neperiano do volume $\left(\mathrm{m}^{3}\right) ; \ln ($ dap $)=\operatorname{logaritmo}$ neperiano do dap $(\mathrm{cm})$.

O modelo de crescimento em volume foi ajustado com os dados de volume (variável y) em cada idade (variável x). A partir do modelo de crescimento em volume foram obtidos os Incrementos Correntes Anuais $\left(\mathrm{ICA}_{\mathrm{v}}\right)$ e os Incrementos Médios Anuais $\left(\mathrm{IMA}_{\mathrm{v}}\right)$ em volume por meio das equações (5) e (6).

$$
\mathrm{ICA}_{\mathrm{V}}=\mathrm{VA}_{(\mathrm{t}+1)}-\mathrm{VA}_{(\mathrm{t})}
$$




$$
\operatorname{IMA}_{\mathrm{v}}=\frac{\mathrm{VA}_{(\mathrm{t})}}{(\mathrm{t})}
$$

Em que: $\mathrm{ICA}_{\mathrm{v}}=$ Incremento corrente anual em volume $\left(\mathrm{m}^{3}\right) ; \mathrm{IMA}_{\mathrm{v}}=$ Incremento médio anual em volume $\left(\mathrm{m}^{3}\right) ; \mathrm{VA}=$ Volume acumulado em diferentes idades durante toda vida da árvore $\left(\mathrm{m}^{3}\right) ; \mathrm{t}=$ Idade em um determinado ano (anos).

\section{Estimativa do Diâmetro Mínimo de Corte e do Ciclo de Corte}

O período preferencial para a colheita da espécie é dado pelos valores de volumes obtidos entre ICAv máximo e IMAv máximo, obtidos por meio das equações 5 e 6 (SCHÖNGART, 2003; MIRANDA et al., 2018). O incremento corrente anual em diámetro ICAd, expressa o crescimento ocorrido entre o início e o fim da estação de crescimento, dado a partir da equação (7).

$$
\mathrm{ICA}_{\mathrm{d}}=\operatorname{dap}_{(\mathrm{t}+1)}-\operatorname{dap}_{(\mathrm{t})}
$$

Em que: ICA $_{d}=$ Incremento corrente anual em diámetro; dap $(\mathrm{t}+1)=$ diâmetro a altura do peito $\left(1,30 \mathrm{~m}\right.$ a partir do nível do solo) no final do período considerado; dap $_{(\mathrm{t})}=$ diâmetro a altura do peito (1,30 $\mathrm{m}$ a partir do nível do solo) no início do período considerado.

Após a definição do DMC, estimou-se o CC a partir da equação (8), o qual foi obtido pelo tempo médio que as espécies levam para passar por classe diamétricas de 10 cm até atingir o DMC específico (SCHÖNGART, 2008).

$$
\mathrm{CC}=\frac{\mathrm{Id}_{(\mathrm{DMC})}}{(\mathrm{DMC} \times 0,1)}
$$

Em que: $\mathrm{CC}=$ Ciclo de Corte $(\operatorname{anos}) ; \mathrm{Id}=$ Idade em anos; $\mathrm{DMC}=$ Diâmetro Mínimo de Corte $(\mathrm{cm})$.

\section{RESULTADOS}

Idade e descrição das amostras 
Qualea paraensis possui anéis de crescimento distintos, a correlação da datação cruzada entre as árvores foi de 0,352 (R-crítico 0,328 a 99\% nível de confiança), permitindo a determinação da idade dessas árvores e o avanço dos estudos de crescimento com aplicações no manejo florestal do Sul da Amazônia. Foram mensurados no total 2.484 anéis de crescimento, e as idades das amostras variam entre 48 e 116 anos, com o diâmetro variando de 35 à $68 \mathrm{~cm}$.

\section{Crescimento da espécie}

Q. paraensis apresentou incremento corrente anual médio de $0,70 \mathrm{~cm}$.ano-1 $( \pm 0,11)$. Seu crescimento inicial é menor, o período de maior crescimento foi entre 23 e 49 anos, voltando a diminuir após esse período (Figura 2). Q. paraensis leva em média 87 anos para chegar ao Diâmetro Mínimo de Corte estabelecido por lei $(50 \mathrm{~cm})$, para o manejo florestal da Amazônia brasileira (Figura 3), evidenciando a necessidade de levar em consideração os diferentes ritmos de crescimento das espécies manejadas.

Figura 2. Variações de incremento em cada idade. Cada linha cinza representa a variação de incremento em diâmetro de uma árvore individual; Linha preta representa a variação do incremento médio quando $n>3$ amostras estavam disponíveis; Linha tracejada vermelha representa o incremento médio anual.

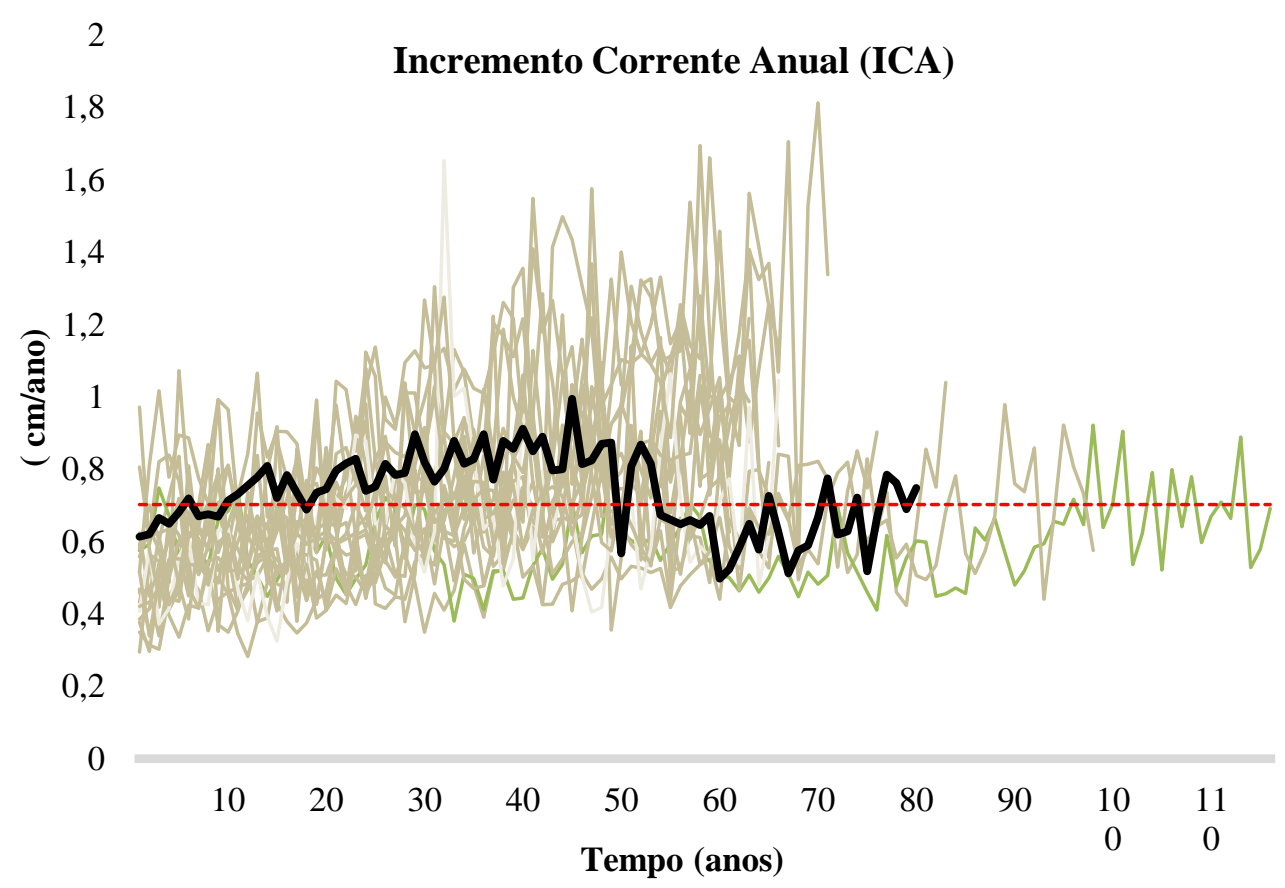

Fonte: Fonte: Os autores (2022). 
Figura 3. Trajetórias de crescimento em diâmetro de Q. paraensis. Linhas cinzas representam a trajetória de crescimento individual da vida de cada árvore; Linha preta mostra o padrão de crescimento médio quando $n>3$ amostras estavam disponíveis; Linha tracejada vermelha indica

o DMC estabelecido pela lei atual (IN 05 de 02/07/2014) para o manejo florestal no Brasil.

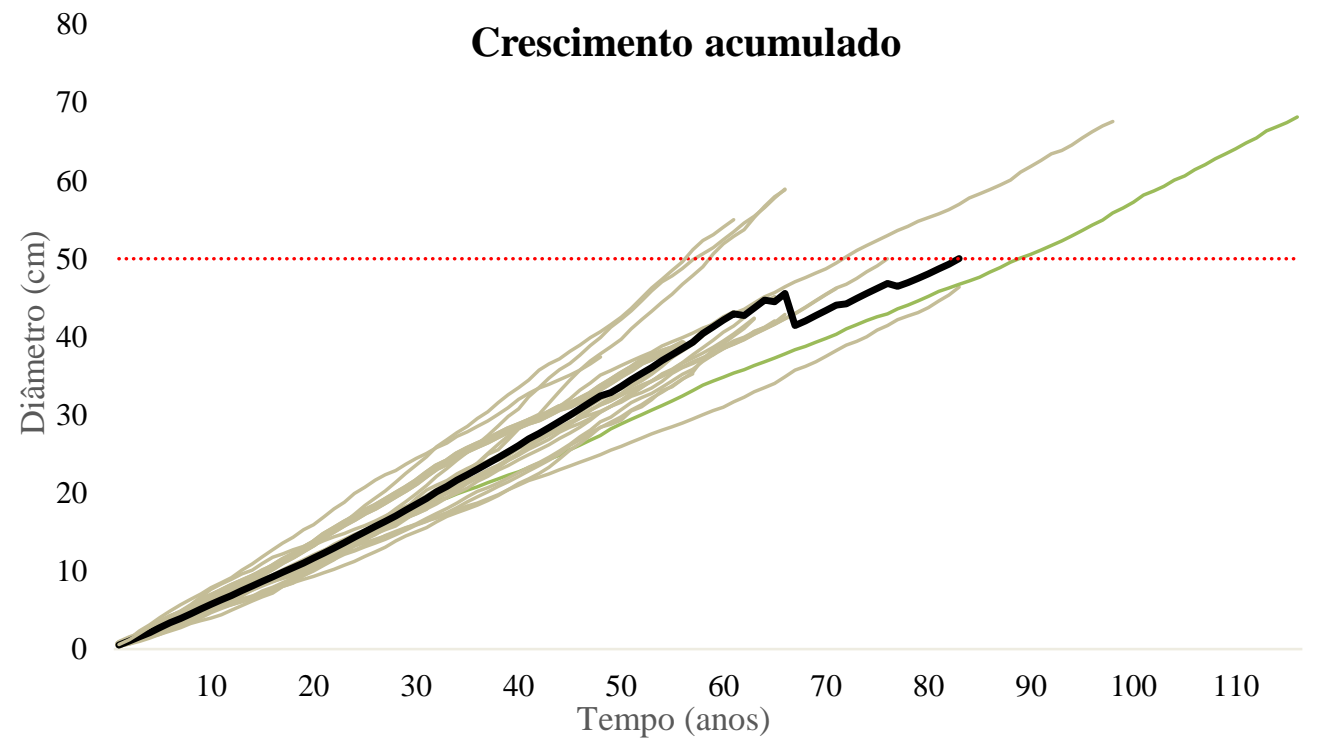

Fonte: Os autores (2022).

\section{Modelagem, Diâmetro Mínimo e Ciclo de Corte}

Foram ajustados os modelos de crescimento em diâmetro e volume, selecionouse as equações que apresentam melhores coeficientes de determinação e erro padrão da estimativa, podem ser vistos na Tabela 2.

Tabela 2. Equações e parâmetros para diâmetro (D) e volume (V) em função da idade (Id) de $Q$. paraensis.

\begin{tabular}{cccc}
\hline Modelo & Equação & $\mathbf{R}^{\mathbf{2}}$ & Syx \\
\hline Ratkowsky & $\mathrm{D}=60,61540379 /\left(1+\mathrm{e}^{(2,3950075-0,046396309 * I d))}\right.$ & 0,98 & 2,47 \\
Gompertz & $\mathrm{V}=7,83918399 * \mathrm{e}^{(-e(1,736210538-0,01872366078 * I d))}$ & 0,99 & 0,14 \\
\hline
\end{tabular}

$\mathrm{R}^{2}=$ Coeficiente de determinação; Syx = Erro padrão da estimativa

A espécie apresentou o pico de incremento em volume ao atingir uma idade de 87 anos (Figura 4). Estimou-se um DMC de aproximadamente $49 \mathrm{~cm}$, e o CC de 17 anos. Os resultados indicam que o CC de 25 a 35 anos exigidos pela legislação atual na Amazônia 
(Instrução Normativa IN 05 de 11/12/2006), não estão de acordo com o ritmo de crescimento de $Q$. paraensis, nessa região, e precisam ser revistos.

Figura 4. Curvas de crescimento a partir das equações ajustadas de diâmetro e volume para $Q$. paraensis. A linha tracejada vermelha indica o DMC estabelecido por lei, a linha tracejada cinza indica o DMC para espécie conforme o estudo.

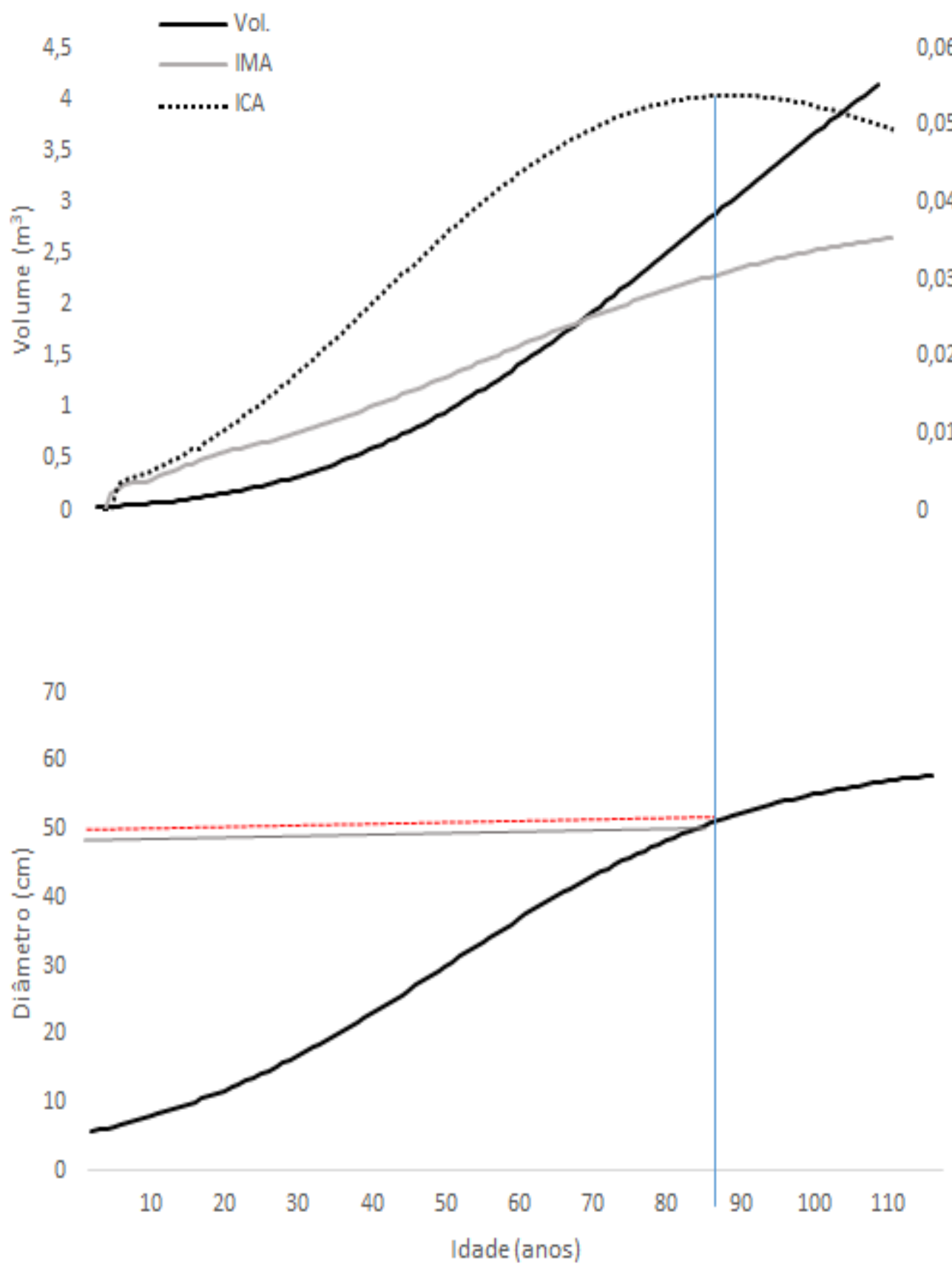

Fonte: Os autores (2022). 


\section{DISCUSSÃO}

\section{Anéis de crescimento de Qualea paraensis}

A acentuada sazonalidade no Mato Grosso, contribui para a distinção de falsos e verdadeiros anéis de crescimento. Mesmo que baixa (0,352), a correlação dos anéis de crescimento ainda foi positiva, possibilitando a determinação da idade das amostras. Miranda et al. (2018) também encontraram uma correlação positiva (R-crítico 0,328 a 99\% nível de confiança) para $Q$. paraensis no Mato Grosso além de evidenciarem a periodicidade anual dos anéis por meio de datação por radiocarbono, assim diminuindo lacunas de estudo para futuros planos de manejo florestal na região. Segundo Brienen et al. (2016), mais de 230 espécies tropicais já tiveram suas periodicidades anuais comprovadas, entre elas: Calophyllum brasiliense (ROSA et al. 2017); Clitoria fairchildiana (XAVIER et al., 2019); e Theobroma cacao (SANTOS et al., 2020), assim auxiliando futuras pesquisas na área.

\section{Crescimento da espécie}

$Q$. paraensis apresentou um incremento médio anual de $0,70 \mathrm{~cm} \cdot a^{-1}$, mantendo um crescimento praticamente constante durante sua trajetória de vida. Implicando diretamente na idade ao se atingir $50 \mathrm{~cm}$ de diâmetro, valor do DMC estabelecido pela legislação atual. A espécie leva em média 87 anos para atingir o DMC atual, mostrando ser uma espécie de rápido crescimento, se comparado com outras espécies Amazônicas, por exemplo, 114 anos para $H$. courbaril e 117 anos para H. serratifolius (ANDRADE, 2015). Existem uma série de fatores que explicam essa variação, tanto características da própria espécie, como fatores ambientais, como por exemplo, a intensidade de luz, a quantidade de água disponível, e agentes fitopatogenicos.

Miranda et al. (2018) avaliaram crescimento em diâmetro de Q. paraensis em uma floresta em segundo ciclo de corte, de terra firme da Amazônia brasileira, e encontraram resultados maiores do que deste estudo, com incremento médio em diâmetro $0,93 \mathrm{~cm}$.ano$1( \pm 0,20)$. Sabe-se que o crescimento da floresta é influenciado pelas condições ambientais como luminosidade e precipitação, uma das hipóteses para a diferença de ritmo de crescimento encontrado por Miranda (2018), é que a área de estudo já tinha uma primeira exploração florestal realizada em 1987, assim aumentando a disponibilidade de recursos que afetam o crescimento.

De qualquer forma, $Q$. paraensis apresentou incrementos altos, se comparado aos demais estudos com espécies amazônicas, Souza (2019) encontrou um incremento de 
0,35 cm.ano ${ }^{-1}$ para Hymenaea courbaril na Flona Tapajós; Em Scleronema micranthum o incremento em diâmetro foi 0,41 cm.ano-1 (SILVA, 2018); Rosa et al. (2017) mostraram 0,43 cm.ano ${ }^{-1}$ para Calophyllum brasiliense; $0,4 \mathrm{~cm}$. ano $^{-1}$ para E. uncinatum (BORGES et al., 2018). Essas diferenças de crescimento entre as espécies mostram a importância da revisão das práticas de manejo adotadas para a Amazônia.

\section{Diâmetro Mínimo e Ciclo de Corte}

Atualmente nos planos de manejo florestal é estabelecido o DMC em $50 \mathrm{~cm}$ e o CC entre 25 e 35 anos para todas as espécies. Para a espécie estudada, é recomendado passar para um CC de 17 anos e o DMC não precisa de alterações. Observa-se que o CC não é estimado pelo tempo total de vida, e sim pelo tempo que a espécie levou para passar por classes diamétricas de $10 \mathrm{~cm}$ até o DMC específico, conforme a equação (7), proposta por Schöngart (2008).

Estudos de crescimentos já são liberados para espécies florestais, porém apenas o Estado do Amazonas já possui uma lei específica para florestas de várzea, proposto pelo estudo de Schöngart (2008). Para esse mesmo autor, espécies de alta densidade, de áreas inundáveis, precisam de um CC de 22 a 32 anos. Outros estudos também apresentam diferentes valores para DMC e CC, como Andrade (2015) que recomenda para $\mathrm{H}$. courbaril o DMC de 62,6 cm e CC de 24 anos. Souza (2019) estimou um DMC de 36,9 cm e CC de 18 anos para Cedrela odorata. Contudo, podem ocorrer variações causadas pelas atividades antropogênicas, como o manejo florestal.

No estudo de Miranda et al., (2018) também foi estimado DMC e CC diferentes da legislação atual, um DMC de aproximadamente $53 \mathrm{~cm}$ e um CC de 11 anos para $Q$. paraensis, em um segundo ciclo de corte. Desta forma, a IN 05 de 11/12/2006 está subestimando o crescimento de espécies individuais, fazendo com que os planos de manejo do estado nem sempre sejam sustentáveis. Contudo, os dois estudos mostram que não são necessários 35 anos para atingir $50 \mathrm{~cm}$ de diâmetro, para Q. paraensis.

\section{CONCLUSÕES}

O crescimento considerado alto de Qualea paraensis deveria ser levado em consideração para as estratégias de manejo a serem adotadas para a espécie. Nos planos de manejo florestal, o Diâmetro Mínimo de Corte de Q. paraensis deve permanecer o mesmo $(50 \mathrm{~cm})$ conforme a legislação atual. Porém, o Ciclo de Corte atualmente utilizado 
nesses planos de manejo não é adequado para $Q$. paraensis em florestas tropicais úmidas de terra firme no norte de Mato Grosso.

Por fim, para que os recursos madeireiros sejam utilizados de forma sustentável, as práticas de manejo para a espécie estudada devem ser revistas e conciliadas com pesquisas da dinâmica florestal para minimizar os impactos ecológicos e garantir futuras explorações.

\section{REFERÊNCIAS}

ANDRADE, V. H. F. Modelos de crescimento para Hymenaea courbaril L. e Handroanthus serratifolius (Vahl) S.O Grose em floresta de terra firme utilizando análise de anéis de crescimento. 2015. 73f. Dissertação (Mestrado em Ciências Agrárias), Universidade Federal do Paraná, Curitiba. 2015.

ARAUJO, R. A.; COSTA, R. B.; FELFILI, J. M.; GONÇALVEZ, I. K.; SOUSA, R. A. T.M. S.; DORVAL, A. Florística e estrutura de fragmento florestal em área de transição na Amazônia Matogrossense no município de Sinop. Acta Amazônica, v. 39, n. 4, p. 865-877, 2009. DOI: https://doi.org/10.1590/S0044-59672009000400015

BORGES, A. L.; MATTOS, P. P.; BRAZ, E. M.; BASSO, A. C. R. Padrão de crescimento de cedrinho em condições de floresta natural no município de Santa Carmem, microregião de Sinop, MT. Embrapa Florestas-Comunicado Técnico (INFOTECA-E), v. 415, 2018.

BRIENEN, J.W.; SCHÖNGART, J.; ZUIDEMA, P.A. Tree Rings in the Tropics: Insights into the Ecology and Climate Sensitivity of Tropical Trees. In: Goldstein G, Santiago LS (ed) Tropical Tree Physiology: Adaptations and Responses in a Changing Environment, Tree Physiology. Springer, 2016. vol 6, p. 439-461.

CIPEM. Centro das Indústrias produtoras e Exportadoras de Madeiras de Mato Grosso. Mato Grosso registraaumento no comércio de produtos florestais. (2012). Disponível em: http://www.cipem.org.br/conteudo.php?sid=44\&cid=1637 Acesso em: 17/08/2020

COLPINI, C.; TRAVAGIN, D.P.; SOARES, T.S.; SILVA, V.S.M. Determinação do volume, do fator de forma e da porcentagem de casca de árvores individuais em uma Floresta Ombrófila Aberta na região noroeste de Mato Grosso. Acta Amazonica, Manaus, v. 39, n.1, p. 97-104, 2009. DOI: http://dx.doi.org/10.1590/S0044$\underline{59672009000100010}$

GROENENDIJK, P.; SASS-KLAASSEN, U.; BONGERS, F.; ZUIDEMA, P.A. Potential of tree-ring analysis in a wet tropical forest: A case study on 22 commercial tree species in Central Africa. Forest Ecology and Management, v.323, p.65-78, 2014. DOI: https://doi.org/10.1016/j.foreco.2014.03.037 
HIGUCHI, N. Utilização e manejo dos recursos madeireiros das florestas tropicais úmidas. Acta Amazonica, v. 24, p. 275-288, 1994. DOI: https://doi.org/10.1590/180943921994243288

LORENZI, H. Árvores brasileiras: manual de identificação e cultivo de plantas arbóreas nativas do Brasil. 2.ed. Editora Instituto Plantarum, Nova Odessa, 2002. v.2. $368 \mathrm{p}$.

MIRANDA, D. L. C.; HIGUCHI, N.; TRUMBORE, S.E.; LATORRACA, J.V. F.; CARMO, J. F.; LIMA, A. J. Using radiocarbon-calibrated dendrochronology to improve tree-cutting cycle estimates for timber management in southern Amazon forests. Trees, v. 32, n. 2, p. 587-602, 2018. DOI: https://doi.org/10.1007/s00468-018$1658-3$

ROSA, S. A.; BARBOSA, A. C. M. C.; JUNK, W. J.; CUNHA, C. N.; PIEDADE, M. T. F.; SCABIN, A. B.; CECCANTINI, G. C. T.; SCHONGART, J. Growth models based on tree-ring data for the Neotropical tree species Calophyllum brasiliense across different Brazilian wetlands: implications for conservation and management. Trees, v. 31, n. 2, p. 729-742, 2017. DOI: https://doi.org/10.1007/s00468-016-1503-5

SANTOS, J. A. R.; LOBÃO, M. S.; ARAUJO, R. M. G. Uso da dendrocronologia para avaliar a produção anual de frutos da espécie theobroma cacao 1. No Sudoeste Da Amazônia. Brazilian Journal of Development, Curitiba, v. 6, n. 7, p. 51001-51011, 2020. DOI: https://doi.org/10.34117/bjdv6n7-662

SECRETARIA DE ESTADO DO MEIO AMBIENTE DE MATO GROSSO - SEMAMT. Beneficiamento e Comércio de Produtos da Madeira por Espécie Florestal. 2016. Disponível em: <http://www.sema.mt.gov.br/portalsisflora/index.php/relatorios>. Acesso em: 16/10/ 2020.

SCHÖNGART, J. Dendrochronologische untersuchungen in überschwemmungswäldern der várzea zentralamazoniens. Goltze, 2003. 149p.

SCHÖNGART, J. Growth-Oriented Logging (GOL): A new concept towards sustainable forest management in Central Amazonian várzea floodplains. Forest Ecology and Management, v. 256, n. 1-2, p. 46-58, 2008. DOI:

https://doi.org/10.1016/j.foreco.2008.03.037

SILVA, F. J. L. Critérios para o manejo florestal de Scleronema micranthum ducke (malvaceae) em uma floresta de terra firme da Amazônia Central. 2018. 39f. Monografia, Universidade do Estado do Amazonas UEA, Itacoatiara, 2018.

SOUZA, A. P.; MOTA, L. L.; ZAMADEI, T.; MARTIM, C. C.; ALMEIDA, F. T.; PAULINO, J. Classificação climática e balanço hídrico climatológico no estado de Mato Grosso. Nativa, v. 1, n. 1, p. 34-43, 2013. DOI: http://dx.doi.org/10.14583/23187670.v01n01a07

SOUZA, L. V. K. S. Dendrocronologia aplicada no manejo de árvores de Cedro (Cedrela odorata) L. e Jatobá (Hymenea coubaril) L. ocorrentes na FLONA Tapajós, PA. 2019. 134f. Tese (Doutorado em Ciências Florestais), Piracicaba, 2019. 
VILANI, M. T.; SANCHES, L.; NOGUEIRA, J. S.; PRIANTE FILHO, F. Sazonalidade da radiação, temperatura e umidade em uma floresta de transição Amazônia Cerrado.

Revista Brasileira de Meteorologia, v. 21, n. 3b, p. 119-131, 2006.

https://doi.org/10.1590/S0102-77862009000300008

WORBES, M. How to measure growth dynamics in tropical trees - a review. IAWA Journal, v. 16, p.337-351, 1995. DOI:_https://doi.org/10.1163/22941932-90001424

WORBES, M. Tree ring analysis. Elsevier, p.1-13, 2004.

XAVIER, C. N.; SOUZA, D. G.; BARBOSA, A. C. M.; SILVA, J. R. M. Tropical dendrochronology applied to invasive tree species in the Brazilian Atlantic Forest.

Journal of Forestry Research, v.2 p. 1-11, 2019. DOI: https://doi.org/10.1007/s11676019-01075-9

Recebido em: 20/01/2022

Aprovado em: 21/02/2022

Publicado em: 24/02/2022 